\section{Fortgeschrittene Basaliome: Wert der Hedgehog-Inhibitoren in Studien bestätigt}

\author{
US-Forscher haben sich einen Überblick über die Datenlage zu den \\ Hedgehog-Inhibitoren verschafft, die zur Therapie von Patienten mit \\ fortgeschrittenen Basalzellkarzinomen zugelassen sind.
}

E ür die Behandlung lokal fortgeschrittener oder metastasierter Basalzellkarzinome stehen in den USA wie in Europa zwei Hemmstoffe des Hedgehog-Signalwegs zur Verfügung: Vismodegib und Sonidegib. Der Hedgehog-Signalweg erfüllt wichtige Aufgaben in der Embryonalentwicklung. Bei Erwachsenen ist er normalerweise inaktiv, eine abnorme Aktivierung findet sich außer bei anderen soliden Tumoren auch beim Basalzellkarzinom.

Eine US-amerikanische Arbeitsgruppe hat sich die Daten der einschlägigen Studien angesehen, die bis zur Zulassung der Substanzen (in Europa für Vismodegib 2013 und für Sonidegib 2015) und später publiziert worden waren. Für eine gepoolte Analyse zu Vismodegib fanden sich acht Untersuchungen mit insgesamt 744 Patienten, davon 704 mit auswertbaren Angaben. Die Rate des vollständigen oder partiellen Therapieansprechens betrug im Durchschnitt $62,1 \%$. Allerdings war hier zu unterscheiden, ob es sich um Patienten mit lokal fortgeschrittenem oder mit gestreu- tem Basalzellkrebs handelte. Von den Ersteren sprachen $64,7 \%$, bei Letzteren $33,6 \%$ auf Vismodegib an. Besonders deutlich war die Differenz mit Blick auf ein vollständiges Ansprechen (31,1 \% bei lokal fortgeschrittenen, 3,9\% bei metastasierten Basaliomen).

Fasste man weniger die Heilung als vielmehr die Kontrolle des Tumorgeschehens in den Blick, so zeigte sich, dass die Therapie bei 33,6\% der Patienten mit lokal fortgeschrittenen Basalzellkarzinomen partiell anschlug und der Krebs bei 27,2\% immerhin stabil blieb. Die entsprechenden Raten für Patienten mit Basalzellkarzinom-Metastasen lagen bei 29,8\% und 49,9\%. Zu berücksichtigen ist, dass die in den einzelnen Studien ermittelten Raten erheblich klafften, für ein Stabilbleiben des Tumors bei lokal fortgeschrittenem Basalzellkrebs beispielsweise zwischen $0,0 \%$ und $48,2 \%$.

Eine gepoolte Datenanalyse wie für Vismodegib war für Sonidegib mangels Masse nicht möglich. Verfügbar waren nur Ergebnisse einer Open-Label-Studie der Phase I mit 16 Patienten und einer multizentrischen, randomisierten und doppelt verblindeten Phase-II-Studie mit relevanten Angaben zu 79 Patienten. Der formale Vergleich mit Vismodegib musste entfallen, wenn auch die berichteten Ansprechraten in etwa die gleiche Größenordnung erreichten.

Die Autoren vermuten, dass sich die Hedgehog-Inhibitoren Vismodegib und Sonidegib in den Wirkungen nicht besonders unterscheiden, weil sie auf einem Gruppeneffekt beruhen. Das wäre insofern plausibel, als beide Moleküle sich gegen den Transmembranrezeptor Smoothened richten. Zudem ähnelt sich das Nebenwirkungsprofil frappant, wobei Muskelspasmen, Alopezie und Schmeckstörungen im Vordergrund stehen. Davon betroffen ist ein erheblicher Anteil der Patienten, die Raten liegen teils über $60 \%$.

Fazit: Zur systematischen Untersuchung von Hedgehog-Inhibitoren bei fortgeschrittenen Basaliomen gab es für Vismodegib ausreichend Studien, um eine gepoolte Analyse vorzunehmen, für Sonidegib hingegen nicht. Vismodegib hatte einen signifikanten und konsistenten Effekt auf die mediane Therapiedauer bei lokal fortgeschrittenen sowie bei metastasierten Basaliomen. Dr. Robert Bublak

Jacobsen AA et al. Hedgehog Pathway Inhibitor Therapy for Locally Advanced and Metastatic Basal Cell Carcinoma. JAMA Dermatol. 2016;152:816-24

\title{
Ein Gerstenkorn ist das jedenfalls nicht!
}

Am rechten Oberlid einer 82-jährigen Frau war es in den vergangenen drei Monaten zu einer rasch zunehmenden Rötung, einer Schwellung und schließlich einer Ulzeration gekommen. Der Hausarzt hatte anfangs, als der Befund noch weniger ausgeprägt war, an ein Gerstenkorn gedacht und mit antibiotischer Salbe behandelt. Der Patientin selbst war das rasche Größenwachstum der Läsion zunächst nicht aufgefallen. Ein Verwandter machte sie schließlich darauf aufmerksam und brachte sie erneut zum Hausarzt, der sie eilig an eine Augenklinik überwies.

Bei Aufnahme zeigte sich eine große, zentral exulzerierte Raumforderung, die fast das gesamte rechte Oberlid einnahm und zur weitgehenden Zerstörung des Oberlidgewebes geführt hatte. Die Histologie ergab ein lokalinvasiv wachsendes Plattenepithelkarzinom. Plattenepithelkarzinome kommen an den Lidern zwar 40-mal seltener vor als Basaliome, sie sind aber wesentlich aggressiver, zerstören rasch die umgebenden Strukturen und erhöhen das Risiko einer lymphatischen regionalen Metastasierung. Junge Frauen schauen vielleicht zu oft in den Spiegel, ältere bisweilen aber zu selten.

Prof.Dr. med. H. S. FüeßI

Hassan A et al. Rapidly enlarging eyelid lesion. BMJ. 2016;352:121

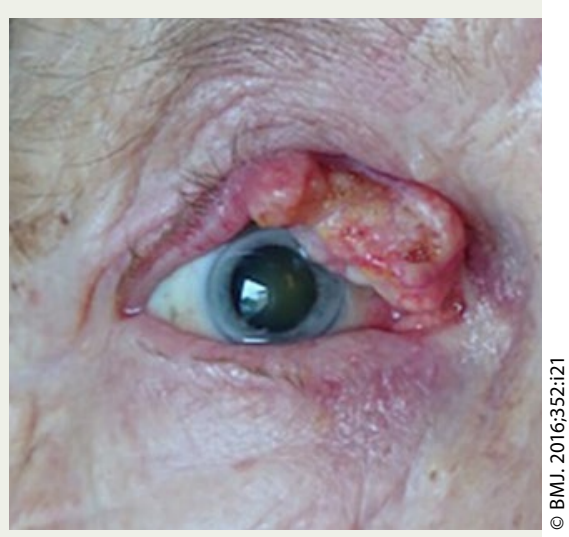

Große, zentral exulzerierte Raumforderung am rechten Augenlid 